Pacific Journal of Mathematics

CONVERGENCE THEOREMS IN BANACH ALGEBRAS 


\section{CONVERGENCE THEOREMS IN BANACH ALGEBRAS}

\section{Robert Beauwens and Jean-Jacques VAn Binnebeek}

It is shown that several convergence theorems for linear operators, usually established by Hilbert space techniques are consequences of the general relations between convergence, monotonicity and order units in ordered Banach spaces.

1. Introduction. Hilbert space techniques have been used to investigate the convergence properties of matrix and linear operator iterative methods by Reich [11], Stein [14], [15], Ostrowski [7], John [6], Householder [4], [5], Petryshyn [8], [9], [10] and de Pillis [2], among others. It is the purpose of the present note to show that these results also follow from the general properties of nonnegative operators on ordered Banach spaces with normal cone and order units. In order to cover most of the above mentioned results without excessive technical preliminaries, we shall present our technique of proof in the framework of Banach algebras.

A direct proof of the relations between convergence, monotonicity and order units, not going through generalizations of the Perron-Frobenius theory, is proposed in $\$ 2$. Needed properties of the hermitian elements of complex unital Banach algebras and of $B^{*}$ algebras are established in $\S 3$. Convergence properties are considered in $\S 4$, mainly in $B^{*}$ algebras.

General notations and terminology are taken from Schaefer [13] with the following exceptions: an element $x$ of an ordered vector space $E$ with cone $C$ is called nonnegative (resp. positive) and denoted $x \geqq 0$ (resp. $x>0$ ) if $x \in C$ (resp. if $x$ is an order unit); $A \in L(E, F)$ where $E$ and $F$ are ordered vector spaces is called nonnegative (resp. positive) if $x \geqq 0$ implies $A x \geqq 0$ (if $x \geqq 0, x \neq 0$ implies $A x>0$ ); it is called monotone if $A x \geqq 0$ implies $x \geqq 0$.

Specific notations and terminology relative to Banach algebras are taken from Bonsall and Duncan [1].

2. Monotonicity and convergence. Convergence properties of nonnegative operators on an ordered (real) Banach space with normal cone and order units are established in the present section. 
THEOREM 2.1. Let $E$ be an ordered (real) Banach space with normal cone and order units; let $T \in \mathscr{L}(E), T \geqq 0$; then

$$
r(T)=\inf _{x>0} \inf _{\alpha>0}\{\alpha \mid T x \leqq \alpha x\}
$$

where $x \in E$ and $\alpha \in R$.

Proof. The relation (2.1) may be written

$$
r(T)=\inf _{x>0}\|T\|_{x}
$$

where $\|T\|_{x}$ denotes the norm induced on $\mathscr{L}(E)$ by the Minkowski functional of the order interval $[-x, x]$; the latter functional is a norm on $E$, equivalent to the original one since the cone of $E$ is normal (cf. Corollary 2 of Theorem 6.2, in Chap. V of Schaefer [13]). Therefore $r(T) \leqq\|T\|_{x}$ for any $x \in E, x>0$; thus

$$
r(T) \leqq \inf _{x>0}\|T\|_{x}
$$

Let $\alpha=r(T)+\epsilon>r(T)$, let $y \in E, y>0$ and set

$$
x=\left(I-\frac{1}{\alpha} T\right)^{-1} y=\sum_{k=0}^{\infty} \frac{1}{\alpha^{k}} T^{k} y
$$

the convergence of the series being guaranteed by $r((1 / \alpha) T)=$ $(1 / \alpha) r(T)<1$; on the other hand

$$
x=y+\sum_{k=1}^{\infty} \frac{1}{\alpha^{k}} T^{k} y \geqq y>0
$$

thus $x>0$; moreover

$$
\left(I-\frac{1}{\alpha} T\right) x=y>0
$$

thus

$$
T x<\alpha x
$$

and, therefore $\|T\|_{x}<\alpha$; since $\alpha$ may be chosen arbitrarily close to $r(T)$, we have 


$$
\inf _{x>0}\|T\|_{x} \leqq r(T)
$$

The conclusion follows.

LEMMA 2.1. Let $E$ be an ordered (real) vector space with order units and let $A \in L(E)$ be monotone and surjective. Then

$$
x>0 \Rightarrow A^{-1} x>0 \text {. }
$$

Proof. Let $x>0, y \in E$ and set $u=A y$. Since $x>0$, there exists $\alpha \in R, \alpha>0$ such that $\alpha u \leqq x$, thus such that $\alpha y \leqq A^{-1} x$. Thus $A^{-1} x$ $>0$.

THEOREM 2.2. Let $E$ be an ordered Banach space with normal cone and order units; let $T \in \mathscr{L}(E), T \geqq 0$. Then the following conditions are equivalent:

(1) $r(T)<1$;

(2) $I-T$ is monotone and surjective;

(3) there exists $x>0$ such that $T x<x$.

Proof.

(1) $\Rightarrow(2)$ is obvious since the cone of $\mathscr{L}(E)$ is closed;

(2) $\Rightarrow$ (3): let $y \in E, y>0$; by Lemma 2.1, we have $x=(I-T)^{-1} y>$ 0 , thus $(I-T) x=y>0$, whence $T x<x$;

(3) $\Rightarrow(1)$ : by Theorem 2.1 .

REMARKs. These results generalize well known theorems of matrix iterative analysis, classically obtained as consequences of the Perron-Frobenius theory of nonnegative matrices. For an intrinsic summary of the theory in finite dimensional spaces, we refer to Rheinboldt and Vandergraft [12]. Note that our attempt here was neither at the most general nor at the most complete possible setting but at the most direct proofs of the few general properties needed in $\$ 4$.

\section{Hermitian elements in Banach algebras.}

3.1. Hermitian elements in complex unital Banach algebras. Let $\boldsymbol{A}$ be a complex unital Banach algebra with unit $e$; by $p(a)$ we denote the functional

$$
p(a)=\sup \{|\lambda| ; \lambda \in V(a)\} \quad a \in A
$$

where $V(a)$ is the numerical range of $a$ in $A$; i.e.

$$
V(a)=\{f(a) ; f \in D(e)\}
$$


with

$$
D(e)=\left\{f \in S\left(A^{\prime}\right) ; f(e)=1\right\}
$$

where $S\left(A^{\prime}\right)$ is the unit sphere in the strong dual $A^{\prime}$ of $A$; by Proposition 10.4 and Theorem 10.14 of Bonsall and Duncan [1], $p(a)$ is a norm on $A$, equivalent to the original one.

An element $a \in A$ is called hermitian if $V(a) \subset R$; the space $E=\operatorname{Her}(A)$ of hermitian elements of $A$ is a real closed subspace of $A$; the set $C=\operatorname{Pos}(A)$ of the elements $a \in A$ such that $V(a) \subset R_{+}$is a proper closed cone in $E$ and we consider here $E$ as an ordered (real) Banach space with nonnegative cone $C$. In particular, we consider that $\mathscr{L}(E)$ is ordered by the induced order (i.e. by the cone of the bounded nonnegative endomorphisms of $E$ ).

Lemma 3.1. Let $A$ be a complex unital Banach algebra, $E=$ $\operatorname{Her}(A), a \in E, a \geqq 0$ and $0 \notin V(a)$; then $a$ is an interior point of $C=\operatorname{Pos}(A)$ in $E$.

Proof. Set $\lambda=\inf \{\alpha \mid \alpha \in V(a)\}$; by Proposition 10.4 of Bonsall and Duncan [1], $\lambda>0$ and

$$
V(a+b) \subset V(a)+V(b) \subset V(a)+[-\|b\|,\|b\|]
$$

for any $b \in E$. Thus, if $\|b\|<\lambda$, we have $V(a+b) \subset R_{+}$, and the proposition follows.

REMARK. When the cone of an ordered topological vector space has nonempty interior, the notions of interior point and of order unit coincide; accordingly for any $a \in \operatorname{Her}(A)$, we have $a>0$ if and only if $f(a)>0$ for any $f \in D(e)$; we also recall here that a cone with order units is generating.

We shall call pencil of elements of an algebra $A$ the set of linear combinations $a-\lambda b$ where $a \in A, b \in A$ and $\lambda$ is a scalar parameter.

Definition 3.1. Let $a$ and $b$ be elements of a complex unital Banach algebra, the set

$$
V_{b}(a)=\{\lambda \mid(\exists f)(f \in D(e) \text { and } f(a-\lambda b)=0)\}
$$

is called the numerical range of the pencil $a-\lambda b$. This notion will be used here under the additional assumption that $b \in \operatorname{Her}(A)$ with $b>0$; in the latter case it is readily seen that 


$$
V_{b}(a)=\left\{\frac{f(a)}{f(b)} ; f \in D(e)\right\}
$$

Definition 3.1 generalizes (3.2) since $V(a)=V_{e}(a)$ where $e$ is the unit of $A$; in other words $V(a)$ is the numerical range of the pencil $a-$

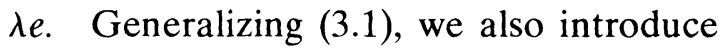

$$
p_{b}(a)=\sup \left\{|\lambda| ; \lambda \in V_{b}(a)\right\}
$$

and we have, in particular, $p(a)=p_{e}(a)$.

LEMMA 3.2. Let $A$ be a complex unital Banach algebra with unit e, and $b \in E=\operatorname{Her}(A), b>0$; then

$$
p_{b}(a)=\inf _{\lambda>0}\{-\lambda b<a<\lambda b\}
$$

Proof. For any $a \in E$ and $\lambda>0$, the relation

$$
-\lambda b<a<\lambda b
$$

is successively equivalent to

$$
\begin{aligned}
& \lambda b+a>0 \text { and } \lambda b-a>0 \\
& f(\lambda b+a)>0 \text { and } f(\lambda b-a)>0 \text { for any } f \in D(e) \\
& \lambda+\frac{f(a)}{f(b)}>0 \text { and } \lambda-\frac{f(a)}{f(b)}>0 \text { for any } f \in D(e) \\
& \left|\frac{f(a)}{f(b)}\right|<\lambda \text { for any } f \in D(e) .
\end{aligned}
$$

Thus (3.7) is equivalent to

(3.13) $p_{b}(a)=\inf \left\{\lambda ;(\forall f)\left(f \in D(e) \Rightarrow\left|\frac{f(a)}{f(b)}\right|<\lambda\right)\right\} \equiv \sup \left\{|\lambda| ; \lambda \in V_{b}(a)\right\}$

which completes the proof.

REMARK. In other words $p_{b}(a)$ is the Minkowski functional of the order interval $[-b, b]$. Since, as stated previously, $p_{e}(a)$ is a norm on $E$, equivalent to $\|a\|$, it follows from the Corollary 2 of V.6.2 in Schaefer [13] that $C=\operatorname{Pos}(A)$ is a normal cone and therefore that $p_{b}(a)$ is also an equivalent norm for arbitrary $b \in E, b>0$. 
It must be remembered that $E$ is considered here as an ordered Banach space; in particular $p_{b}(a)$ is not an algebra norm. Nevertheless, we have

Lemma 3.3. Let $A$ be a complex unital Banach algebra, $E=$ $\operatorname{Her}(A), a \in A, b \in E, b>0$. Then

$$
r\left(b^{-1} a\right) \leqq p_{b}(a) \leqq\left\|b^{-1}\right\|\|a\| .
$$

Proof. If $\lambda \in \sigma\left(b^{-1} a\right)$, then $a-\lambda b$ is singular, thus $0 \in$ $\sigma(a-\lambda b) \subset V(a-\lambda b)$; therefore, for some $f \in D(e)$, we have $f(a-\lambda b)=0$, thus also $\lambda=f(a) / f(b)$, i.e. $\lambda \in V_{b}(a)$. The left inequality follows.

The right inequality follows from

$$
p_{b}(a) \leqq \frac{\sup \{|\lambda| ; \lambda \in V(a)\}}{\inf \{\lambda ; \lambda \in V(b)\}}
$$

since

$$
\sup \{|\lambda| ; \lambda \in V(a)\} \leqq\|a\|
$$

and

$$
\inf (V(b))=\inf (\sigma(b))=\frac{1}{\sup \left(\sigma\left(b^{-1}\right)\right)}=\frac{1}{\left\|b^{-1}\right\|}
$$

by Theorems 10.4, 10.5, 10.17 and 7.4 of Bonsall and Duncan [1].

3.2. The Stein transformation in $B^{*}$ algebra. We recall that a $B^{*}$ algebra is a complex unital Banach algebra $A$ with a linear involution $a \rightarrow a^{*}$ such that $\left\|a^{*} a\right\|=\|a\|^{2}$ for any $a \in A$. If $A$ is a $B^{*}$ algebra, $a \in A$ is hermitian if and only if it is symmetric, i.e. $a^{*}=a$.

Definition 3.1. Let $A$ be a $B^{*}$ algebra and let $a \in A$; then the linear transformation $T_{a}$ on $E=\operatorname{Her}(A)$ into $A$ defined by $T_{a}: x \rightarrow a^{*} x a$ for each $x \in E$ is called the Stein transformation associated with $a$.

Lemma 3.4. Let $A$ be $a B^{*}$ algebra, $E=\operatorname{Her}(A)$ and $T_{a}$ be the Stein transformation associated with $a \in A$; then $T_{a} \in \mathscr{L}(E)$ and $T_{a} \geqq 0$.

Proof. Let $x \in E, x \geqq 0$ and $y=T_{a}(x)=a^{*} x a$; by Lemma 38.7 of Bonsall and Duncan [1], there exists $u \in E, u \geqq 0$ such that $u^{2}=x$; since $E=\operatorname{Sym}(A)$ we have $u^{*}=u$, thus $y=\left(a^{*} u^{*}\right)(u a)$ which entails $y \geqq 0$ 
by Lemma 38.9 of Bonsall and Duncan [1]. On the other hand, it is clear that $T_{a}$ is bounded.

Lemma 3.5. Let $A$ be a $B^{*}$ algebra with unit e, $E=\operatorname{Her}(A)$, and $T_{a}$ be the Stein transformation associated with $a \in A$. Then

(1) $\left\|T_{a}\right\|=\|a\|^{2}$

(2) $\quad r\left(T_{a}\right)=(r(a))^{2}$.

Proof. We have

$$
\left\|T_{a}\right\|=\sup _{\substack{x \in E \\ x \neq 0}} \frac{\left\|a^{*} x a\right\|}{\|x\|} \leqq\left\|a^{*}\right\| \cdot\|a\|=\|a\|^{2}
$$

Moreover, for $x=e$,

$$
\left\|T_{a}\right\| \geqq\left\|a^{*} a\right\|=\|a\|^{2}
$$

and the first assertion follows.

Applying (1) to $a^{n}$, we get

$$
\left\|T_{a}^{n}\right\|=\left\|T_{a^{n}}\right\|=\left\|a^{n}\right\|^{2}
$$

Therefore

$$
r\left(T_{a}\right)=\lim _{n \rightarrow \infty}\left\|T_{a}^{n}\right\|^{1 / n}=\lim _{n \rightarrow \infty}\left\|a^{n}\right\|^{2 / n}=(r(a))^{2} .
$$

4. Convergence theorems. New proofs, based on the relations between monotonicity, convergence and order units are proposed in the present section for several convergence theorems usually established in the framework of Hilbert spaces. [4]:

The following theorem is a generalization of a result by Householder

THEOREM 4.1. Let $A$ be a complex unital Banach algebra with unit $e, E=\operatorname{Her}(A)$ and $t \in E$; then $r(t)<1$ if and only if $-e<t<e$.

Proof. By Lemma 3.3 and by Theorem 10.17 of Bonsall and Duncan [1], we have $r(t)=p(t)$. The conclusion follows by Lemma 3.2.

We now consider the application of Theorem 2.2 in $B^{*}$ algebras; thus we have

Theorem 4.2. Let $A$ be a $B^{*}$ algebra, $E=\operatorname{Her}(A)$ and $T_{t}$ be the 
Stein transformation associated with $t \in A$; then the following conditions are equivalent:

(1) $r(t)<1$;

(2) $I-T_{t}$ is monotone and surjective;

(3) there exists $x \in E, x>0$ such that $\left(I-T_{t}\right) x>0$.

Proof. By Lemma 3.4, $T_{t} \in \mathscr{L}(E)$ with $T_{t} \geqq 0$; by Lemma 3.5, $r\left(T_{t}\right)=r(t)^{2}$; the conclusion follows by Theorem 2.2.

As a consequence, we have the following generalization of a theorem by Stein [14], [15].

Theorem 4.3. Let $A$ be $a B^{*}$ algebra, $E=\operatorname{Her}(A), a \in E$ and $T_{t}$ be the Stein transformation associated with $t \in A$. If $\left(I-T_{t}\right) a>0$, then $r(t)<1$ if and only if $a>0$.

Proof. Let $b=\left(I-T_{t}\right) a>0$. If $r(t)<1, I-T_{t}$ is monotone and surjective, thus $a>0$ by Lemma 2.1. If, on the other hand, we have $a>0$, then $r(t)<1$ by Theorem 4.2, [3].

The preceding results apply in practice when, in a given Banach space $V$, one solves a linear system

$$
a x=y, \quad x \in V, \quad y \in V, \quad a \in \mathscr{L}(v)
$$

by an iterative scheme of the form

$$
b x^{(j+1)}=c x^{(j)}+y \quad j=0,1,2 \cdots
$$

with $a=b-c$; assuming that $b^{-1} \in \mathscr{L}(V)$, one introduces the iteration operator $t=b^{-1} c$ and the condition $r(t)<1$ is equivalent to the normconvergence of the Neumann expansion for $(e-t)^{-1}$ where $e$ is the identity operator of $\mathscr{L}(V)$.

In this context, we have the following generalization of Theorem 4.1 (cf. Petryshyn [9]):

Theorem 4.4. Let $A$ be $a B^{*}$ algebra and $E=\operatorname{Her}(A)$; let $a, b, c \in E$ with $a=b-c, b>0$ and set $t=b^{-1} c$. Then $r(t)<1$ if and only if $-b<c<b$.

Proof. Since $b \in E, b>0$, there exists $u \in E, u>0$ such that $u^{2}=b$. Let $T_{u^{-1}}$ be the Stein transformation associated with $u^{-1}$ and $S_{u^{-1}}$ be the similarity transformation defined by $S_{u^{-1}} x=u x u^{-1}$ for all $x \in A$. We have 


$$
T_{u^{-1}}(b \pm c)=S_{u^{-1}}(e \pm t)
$$

thus, by Lemma 3.4, $S_{u^{-1}} t \in E$ and, by Theorem 4.1, $r(t)=r\left(S_{u^{-1}} t\right)<1$ if and only if

$$
-e<S_{u^{-1}} t<e
$$

or

$$
S_{u^{-1}}(e \pm t)>0
$$

Since, on the other hand, $T_{u^{-1}}$ is both nonnegative and monotone with $T_{u}=\left(T_{u^{-1}}\right)^{-1}$, it follows from Lemma 2.1 that the preceding relation is equivalent to

$$
b \pm c=T_{u} S_{u^{-1}}(e \pm t)>0
$$

or

$$
-b<c<b \text {. }
$$

As an immediate consequence, we have the following result in $B^{*}$ algebras.

Corollary. Let $A$ be $a B^{*}$ algebra, $E=\operatorname{Her}(A), a, b \in E, b>0$; then $r\left(b^{-1} a\right)=p_{b}(a)$.

REMARK. It follows from Lemma 3.3 that the sufficient condition of Theorem 4.4 still holds in a complex unital Banach algebra; whether or not the necessary condition also extends in the latter case remains an open question. In the same framework, practical application of Theorem 4.3 is made easier by the following result which generalizes a theorem by Feingold and Spohn [3].

Theorem 4.5. Let $A$ be $a B^{*}$ algebra and $E=\operatorname{Her}(A)$; let $a \in E$, $b, c \in A$ with $a=b-c$ and $b \in \operatorname{Inv}(A)$; set $t=b^{-1} c$ and let $T_{t}$ be the Stein transformation associated with $t$. Then $\left(b^{*}+c\right) \in E ;$ moreover $\left(I-T_{t}\right) a>0$ if and only if $\left(b^{*}+c\right)>0$.

Proof. We have

$$
\left(b^{*}+c\right)^{*}=b+c^{*}=a+c+c^{*}=a^{*}+c+c^{*}=b^{*}+c
$$

thus $\left(b^{*}+c\right) \in E$. 
On the other hand, the following identity is readily established

$$
\left(I-T_{t}\right) a=a b^{*-1}\left(b^{*}+c\right) b^{-1} a
$$

and the conclusion follows by Lemma 3.4 and Lemma 3.1.

As a consequence, we have the following generalization of a theorem of matrix iterative analysis, sometimes attributed to Reich [11] and Ostrowski [7] who considered the particular case of the successive overrelaxation method; its general form seems however to appear first in John [6] for the sufficient condition, and in Householder [5].

THEOREM 4.6. Let $A$ be $a B^{*}$ algebra and $E=\operatorname{Her}(A)$; let $a \in E$, $b, c \in A$ with $a=b-c$ and $b \in \operatorname{Inv}(A)$, and set $t=b^{-1} c$. If $\left(b^{*}+c\right)>$ 0 , then $r(t)<1$ if and only if $a>0$.

Proof. In virtue of Theorem 4.5, the proposition is a restatement of Theorem 4.3.

With slight additional notations, these results also cover generalizations of Petryshyn's theorems for bounded operators.

TheOrem 4.7. Let $A$ be $a B^{*}$ algebra and $E=\operatorname{Her}(A)$; let $a, b, c, k \in A$ with $a=b-c, k \in \operatorname{Inv}(A), k a$ and $k b \in E, k b>0$, and set $t=b^{-1} c$. Then $r(t)<1$ if and only if $-k b<k c<k b$.

Proof. Using the notations of the proof of Theorem 4.4, but with $u^{2}=k b$, we have

$$
T_{u^{-1}}(k b \pm k c)=S_{u^{-1}}(e \pm t)
$$

The conclusion follows, as precedingly.

Remark. When $k \in E$ with $k>0$, an element $a \in A$ such that $k a \in E$ is termed $k$-symmetric by Petryshyn; it is termed $k$-positivedefinite if, in addition, $k a>0$. In the case of finite matrices, alternate characterizations of $k$-positive-definite elements are considered in [8].

On the other hand, Theorems 4.5 and 4.6 admit the following generalizations.

THEOREM 4.8. Let $A$ be $a B^{*}$ algebra and $E=\operatorname{Her}(A)$; let $a, b, c, k, l \in A, a=b-c, k a l \in E, b, k, l \in \operatorname{Inv}(A)$; set $u=l^{-1} b^{-1} c l$ and let $T_{u}$ be the Stein transformation associated with $u$. Then $l^{*} b^{*} k^{*}+k c l \in$ $E$ and $\left(I-T_{u}\right) k a l>0$ if and only if $l^{*} b^{*} k^{*}+k c l>0$. 
Proof. We have

$$
\begin{aligned}
\left(l^{*} b^{*} k^{*}+k c l\right)^{*} & =k b l+l^{*} c^{*} k^{*} \\
& =k a l+k c l+l^{*} c^{*} k^{*} \\
& =l^{*} a^{*} k^{*}+k c l+l^{*} c^{*} k^{*} \\
& =l^{*} b^{*} k^{*}+k c l
\end{aligned}
$$

thus $l^{*} b^{*} k^{*}+k c l \in E$.

On the other hand, we have

$$
\left(I-T_{u}\right) k a l=l^{*} a^{*} b^{*-1} l^{*-1}\left(l^{*} b^{*} k^{*}+k c l\right) l^{-1} b^{-1} a l
$$

and the conclusion follows by Lemma 3.4 and Lemma 3.1 .

THEOREM 4.9. Let $A$ be $a B^{*}$ algebra and $E=\operatorname{Her}(A)$; let $a, b, c, k, l \in A, a=b-c, k a l \in E, b, k, l \in \operatorname{Inv}(A)$ and set $t=b^{-1} c$. If $l^{*} b^{*} k^{*}+k c l>0$, then $r(t)<1$ if and only if $k a l>0$.

Proof. If $u=l^{-1} t l$, we have $r(t)=r(u)$; therefore, in virtue of Theorem 4.8, the proposition is a restatement of Theorem 4.3.

\section{REFERENCES}

1. F. F. Bonsall and J. Duncan, Complete Normed Algebras, Springer-Verlag, Berlin (1973).

2. J. de Pillis, Gauss-Seidel convergence for operators on Hilbert space, SIAM J. Numer. Anal., 10 (1973), 112-122.

3. D. Feingold and D. Spohn, Un théorème simple sur les normes de matrices et ses conséquences,

C. R. Acad. Sci. Paris, 256 (1963), 2758-2760.

4. A. S. Householder, On the convergence of matrix iterations, Rep. ORNL-1883, Oak Ridge National Laboratory (1955).

5. - The approximate solution of matrix problems, J. Assoc. Comp. Mach., 5 (1958), 205-243.

6. F. John, Lectures on advanced numerical analysis, Gordon and Breach (1967), from mimeographed lectures notes, New York University (1956).

7. A. M. Ostrowski, On the linear iteration procedures for symmetric matrices, Rend. Mat. e Appl., 14 (1954), 140-163.

8. W. V. Petryshyn, On the generalized overrelaxation method for operator equations, Proc. Amer. Math. Soc., 14 (1963), 917-924.

9. - On the extrapolated Jacobi or simultaneous displacements method in the solution of matrix and operator equations, Math. Comp., 19 (1965), 37-55.

10. - Remarks on the generalized overrelaxation and the extrapolated Jacobi methods for operator equations in Hilbert space, J. Math. Anal. Appl., 29 (1970), 558-568.

11. E. Reich, On the convergence of the classical iterative method of solving linear simultaneous equations, Ann. Math. Statist., 20 (1949), 448-451.

12. W. C. Rheinboldt and J. Vandergraft, A simple approach to the Perron Frobenius theory for positive operators on general partially-ordered finite-dimensional linear spaces, Math. Comp., 27 (1973), 139-145. 
13. H. H. Schaefer, Topological Vector Spaces, Springer-Verlag, Berlin (1966).

14. P. Stein, The convergence of Seidel iterants of nearly symmetric matrices, M.T.A.C., 5 (1951), 237-240.

15. P. Stein, Some general theorems on iterants, J. Res. Nat. Bur. Stand., 48 (1952), 82-83.

Received June 3, 1975. Partly supported by the Fonds National de la Recherche Scientifique of Belgium.

UNIVERSITÉ LIBRE DE BRUXELLES 



\section{Pacific Journal of Mathematics}

Vol. 68, No. 1

March, 1977

Richard Julian Bagby, On $L^{p}, L^{q}$ multipliers of Fourier transforms . .......

Robert Beauwens and Jean-Jacques Van Binnebeek, Convergence theorems in

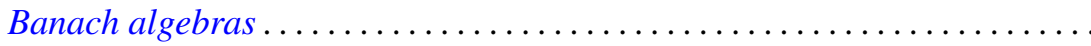

James Cyril Becker, Skew linear vector fields on spheres in the stable

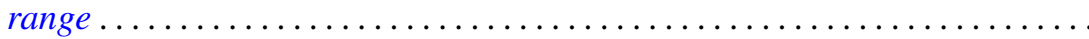

Michael James Beeson, Continuity and comprehension in intuitionistic formal

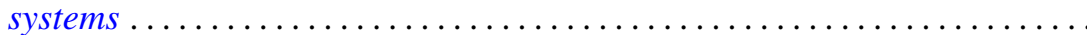

James K. Deveney, Generalized primitive elements for transcendental field

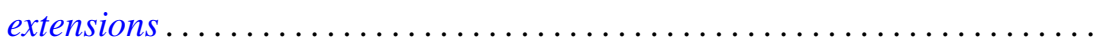

Samuel S. Feder, Samuel Carlos Gitler and K. Y. Lam, Composition properties

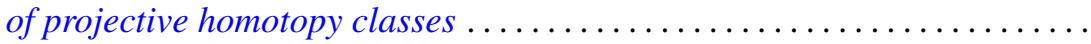

Nathan Jacob Fine, Tensor products of function rings under composition ......

Benno Fuchssteiner, Iterations and fixpoints . . . . . . . . . . . . . .

Wolfgang H. Heil, On punctured balls in manifolds

Shigeru Itoh, A random fixed point theorem for a multivalued contraction

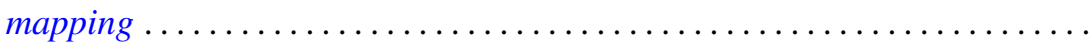

Nicolas P. Jewell, Continuity of module and higher derivations . . . . . . ......

Roger Dale Konyndyk, Residually central wreath products . . . . . . . . . . .

Linda M. Lesniak and John A. Roberts, On Ramsey theory and graphical

parameters.

Vo Thanh Liem, Some cellular subsets of the spheres.

Dieter Lutz, A perturbation theorem for spectral operators

P. H. Maserick, Moments of measures on convex bodies ... . . .

Stephen Joseph McAdam, Unmixed 2-dimensional local domains . .

D. B. McAlister and Norman R. Reilly, E-unitary covers for inverse semigroups...

William H. Meeks, III and Julie Patrusky, Representing codimension-one

homology classes by embedded submanifolds . . .

Premalata Mohapatro, Generalised quasi-Nörlund summability . .

Takahiko Nakazi, Superalgebras of weak-*Dirichlet algebras .

Catherine Louise Olsen, Norms of compact perturbations of operators .

William Henry Ruckle, Absolutely divergent series and isomorphism of

subspaces. II.

Bernard Russo, On the Hausdorff-Young theorem for integral operators .

Arthur Argyle Sagle and J. R. Schumi, Anti-commutative algebras and

homogeneous spaces with multiplications ............

Robert Evert Stong, Stiefel-Whitney classes of manifolds .

D. Suryanarayana, On a theorem of Apostol concerning Möbius functions of

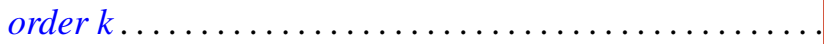

Yoshio Tanaka, On closedness of $C$ - and $C^{*}$-embeddings . . 